\title{
Mécanismes de la contractilité cardiaque
}

On connaît aujourd'hui les mécanismes physico-chimiques de la contractilité des fibres du myocarde. Leur altération peut être à l'origine des défaillances cardiaques.

\section{Philippe Lechat}

Chef de clinique-assistant, service de cardiologie, Chu Pitié-Sal pêtrière.

\section{Michel Eugène}

Chef de travaux, assistant, service d'explorations fonctionnelles, Chu Pitié-Salpêtrière.

\section{Michel Komajda}

Assistant des hôpitaux. Service de cardiologie, Chu Pitié-Salpêtrière.

\section{ADRESSE}

Ph. Lechat, M. Eugène, M. Komajda :Chu Pitié-Salpêtrière, service de cardiologie, 47, Boulevard de l'Hôpital, 75651 Paris' Cedex 13. e calcium joue un rôle primordial dans le couplage des phénomènes d'excitation-contraction-relaxation.

A la suite de l'excitation membranaire, l'élévation de la concentration calcique cytosolique déclenche la contraction par interaction entre les filaments d'actine et de myosine. La relaxation est secondaire au recaptage du calcium par le réticulum sarcoplasmique et par les systèmes d'expulsion calcique vers le milieu extracellulaire. La régulation de l'inotropisme met en jeu de nombreux systèmes : canaux calciques, systèmes d'échanges $\mathrm{Na}-\mathrm{Ca}$, calmoduline et enfin protéines kinases qui régulent l'activité des systèmes enzymatiques de transport calcique intracellulaires et transmembranaires ainsi que l'activité des protéines contractiles.

\section{Excitation membranaire, influx calcique}

Schématiquement, l'excitation membranaire déclenche le potentiel d'action: la phase zéro correspond à une entrée rapide dépolarisante d'ions sodium $(\mathrm{Na})$, la phase I à un courant partiellement repolarisant, la phase 2 à une entrée d'ions calcium et sodium par ouverture des canaux du courant calcico-sodique (courant lent), la phase 3 à une sortie repolarisante d'ions potassiques vers le milieu extracellulaire.

Ces phénomènes membranaires entrainent une augmentation de la concentration calcique cytosolique qui passe de $10^{-7} \mathrm{M}$ au repos à I0 $\mathrm{M}$ lors de la contraction. Or, comme l'ont bien établi les expériences de voltage clamp $[\mathrm{I}, 2]$, la quantité de calcium fournie par le courant calcique lent n'est pas suffisante pour provoquer la contraction musculaire, sauf chez la grenouille [3]. Ce fait est d'autant plus paradoxal que le calcium extracellulaire est strictement indispensable à la contraction cardiaque par opposition à celle du muscle strié squelettique [4]. L'influx calcique du courant lent induit la libération d'un pool calcique intracellulaire. Le réticulum sarcoplasmique en est la localisation principale comme l'ont bien montré des études utilisant les radio-isotopes et les préparations "fibres cardiaques pelées" sans sarcolemme $[5,6]$. Le mécanisme de ce phénomène appellé calcium induced calcium release, ou libération du calcium induite par le calcium, reste inconnu. D'après Lullman [7], le principal site intracellulaire du pool calcique mobilisable serait le versant interne du sarcolemme (sur les résidus phosphatidyl sérine des phospholipides). En fait, les deux localisations du pool calcique intracellulaire : réticulum sarcoplasmique et sarcolemme, ne s'excluent pas mutuellement. L'importance relative du courant lent et des pools calciques intracellulaires varie selon les espèces et selon le degré de maturation. Ainsi, le myocarde de grenouille est-il dépourvu de réticulum sarcoplasmique (ou presque) et c'est le courant lent qui fournit l'essentiel du calcium pour la contraction. C'est l'inverse chez le rat adulte dont le réticulum sarcoplasmique est très développé avec un potentiel d'action triangulaire presque totalement dépourvu de courant lent.

L'élévation de la concentration cal- 
cique intracellulaire induit l'interpénétration des filaments d'actine et de myosine à l'origine de la contraction. Le filament fin comporte trois types de protéines différentes : l'actine, la troponine et la tropomyosine. La troponine comprend ellemême trois sous-unités : la troponine $C$ qui fixe le calcium, la troponine I qui inhibe l'interaction ATPasique de la myosine, et la troponine $T$ qui se fixe à la tropomyosine. L'actine présente deux filaments formant une double hélice, dans le sillon de laquelle se trouvent des molécules de tropomyosine unies bout à bout et qui confèrent aux filaments fins leur rigidité.

La myosine est une molécule hexamère formée par l'association d'une paire de chainnes lourdes et de deux paires de chaînes légères. C'est une molécule asymétrique présentant une partie fibrillaire ou queue et une partie globulaire ou tête qui possède le site actif de fixation de l'actine et le site actif de l'ATPase. Cette enzyme présente trois formes isozymiques $V_{1} V_{2} V_{3}$ qui possèdent des activités ATPases différentes ( $\mathrm{V}_{\mathrm{I}}>\mathrm{V}_{3}$ ), (Voir page 66 l'article de Ketty Schmartz).

Les phénomènes mécaniques de la contraction sont déclenchés par la fixation du calcium sur la troponine C. La molécule de tropomyosine subit alors un changement conformationnel, ce qui induit la fixation de l'actine à la myosine. L'interaction actine-myosine peut alors se produire avec hydrolyse concomitante de l'ATP par l'ATPase de la myosine. Le cycle d'interaction comporte 4 phases :

(a) attachement de la myosine et de l'actine. La myosine porte sur son site ATPase : ADP et $\mathrm{P}$, les produits de l'hydrolyse de l'ATP;

(b) inclinaison de la tête de la myosine qui réalise le déplacement de la molécule d'actine à laquelle elle est liée, avec, parallèlement, départ d'ADP et $\mathrm{P}$;

(c) détachement de la tête de la myosine de l'actine et fixation d'ATP sur son site ATPasique;

(d) retour de la tête de la myosine à sa position de départ avec hydrolyse de l'ATP, la tête se trouvant alors devant le site suivant de l'actine.

L'hydrolyse de l'ATP est donc nécessaire au retour de la tête de sa position "inclinée " à la position "droite ". Le nombre de cycles association-dissociation de l'actine et de la myosine (cycling cross bridges) qui détermine la tension exercée par la fibre dépend de la concentration en $\mathrm{Ca}^{++}$du cytosol et du temps pendant lequel le $\mathrm{Ca}^{++}$reste fixé à la troponine $\mathrm{C}$.

La tension développée dépend de nombreux facteurs : hypoxie, ischémie, acidose, augmentation de la concentration en $\mathrm{Mg}^{++}$, phosphorylation de la troponine I, qui la font diminuer en réduisant la sensibilité au calcium. La phosphorylation de la TI peut être effectuée par une kinase activée par l'AMP cyclique (ce qui favorise l'accélération de la relaxation observée avec la stimulation béta-adrénergique par exemple).

Enfin, au cours de la concentration, le calcium, outre sa fixation sur la troponine $C$ va également former un complexe avec une protéine cytosolique, la calmoduline. Le complexe ainsi formé stimule de nombreuses activités enzymatiques : d'une part, les protéines kinases responsables de la phosphorylation du phospholambdan, du métabolisme du glycogène (phosphorylase et glycogène synthétase), d'autre part l'ATPase $\mathrm{Ca}-\mathrm{Mg}$ dépendante du sarcolemme, l'adényl cyclase et la phosphodiestérase contrôlant le taux d'AMP cyclique intracellulaire.

\section{Relaxation. repolarisation}

La relaxation survient lorsque la concentration du $\mathrm{Ca}^{++}$près des myofilaments est réduite par le repompage actif de celui-ci par le réticulum sarcoplasmique, grâce à une ATPase $\mathrm{Ca}-\mathrm{Mg}$ dépendante. Cette enzyme possède une très haute affinité pour le calcium, ce qui permet une grande vitesse de fixation et de transport. Elle est activée par le phospholambdan, protéine du réticulum sarcoplasmique agissant lorsqu'elle est phosphorylée par une kinase cellulaire AMP cyclique-dépendante et stimulée par la calmoduline. Ainsi la relaxation peut-elle être accélérée par toute augmentation du taux d'AMPc (stimulation des récepteurs bétaadrénergiques, figure I, inhibition de la phosphodiestérase). Enfin les systèmes de pompe à calcium du sarcolemme (ATPases $\mathrm{Mg}-\mathrm{Ca}$ dépendantes) participent également à l'expulsion calcique pendant la relaxation. Leur activité est stimulée par la calmoduline. A noter qu'il existe également des flux calciques de part et d'autre de la membrane interne des mitochondries grâce à des systèmes de transport indépendants. Leur rôle dans la régulation des phénomènes de contractionrelaxation reste mal connu.

L'affinité du calcium pour la troponine $\mathrm{C}$ module également la relaxation. Elle augmente avec la charge exercée sur la fibre. Ces deux phénomènes (recaptage par le réticulum sarcoplasmique et variation d'affinité du calcium pour la troponine C) sont à l'origine de la dépendance de la relaxation vis-à-vis de la charge [8] : la relaxation est d'autant plus tardive et sa vitesse d'autant plus faible que la charge est plus grande et le raccourcissement plus faible. Ce phénomène dit de load-dependence (dépendance de la charge) n'existe pas chez la grenouille dont le myocarde est dépourvu de réticulum sarcoplasmique. Il est également supprimé lors de l'ischémie, qui altère le recaptage calcique par le réticulum sarcoplasmique.

Les substances inhibant la phosphodiestérase accélèrent la relaxation en augmentant le taux d'AMPc intracellulaire. Cependant, aux fortes concentrations, certaines d'entre elles (bases xanthiques, amrinone) la réduisent en augmentant l'affinité du calcium pour la troponine $C$ et en ralentissant les pompes à calcium du réticulum sarcoplasmique [9].

\section{Restauration de I'homéostasie}

Après le potentiel d'action, la restauration des concentrations ioniques intracellulaires $(\mathrm{Na}, \mathrm{K}, \mathrm{Ca})$ doit s'effectuer afin de maintenir l'homéostasie intracellulaire. La " pompe à sodium, système enzymatique ATPasique, expulse de la cellule $3 \mathrm{Na}$ et fait rentrer deux ions potassiques, déséquilibre à l'origine de la polarisation membranaire (figure 2).

Quant au surplus calcique, il est expulsé vers le milieu extracellulaire 


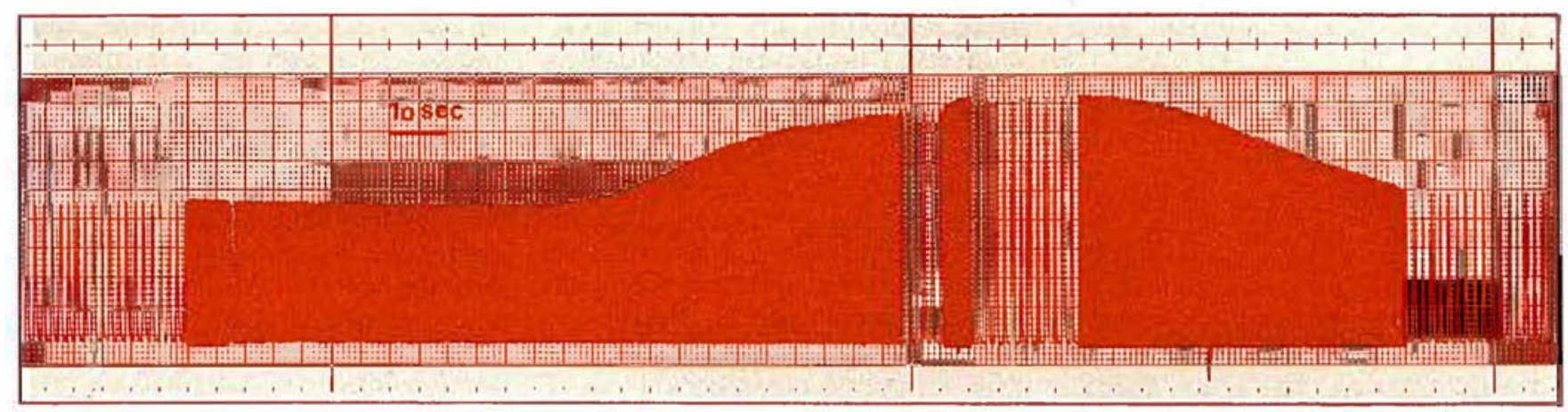

Figure I. Effets inotropes de l'isoprénaline (agoniste bêta-adrénergique). L'effet inotrope ne s'accompagne pas d'élévation de la tension diastolique, par stimulation du recaptage du calcium par le reticulum sarcoplasmique. (même préparation que figure 3 ).

Figure 2. Représentation des différents systèmes intervenant dans la régulation des phénomènes d'excitation-contraction-relaxation.

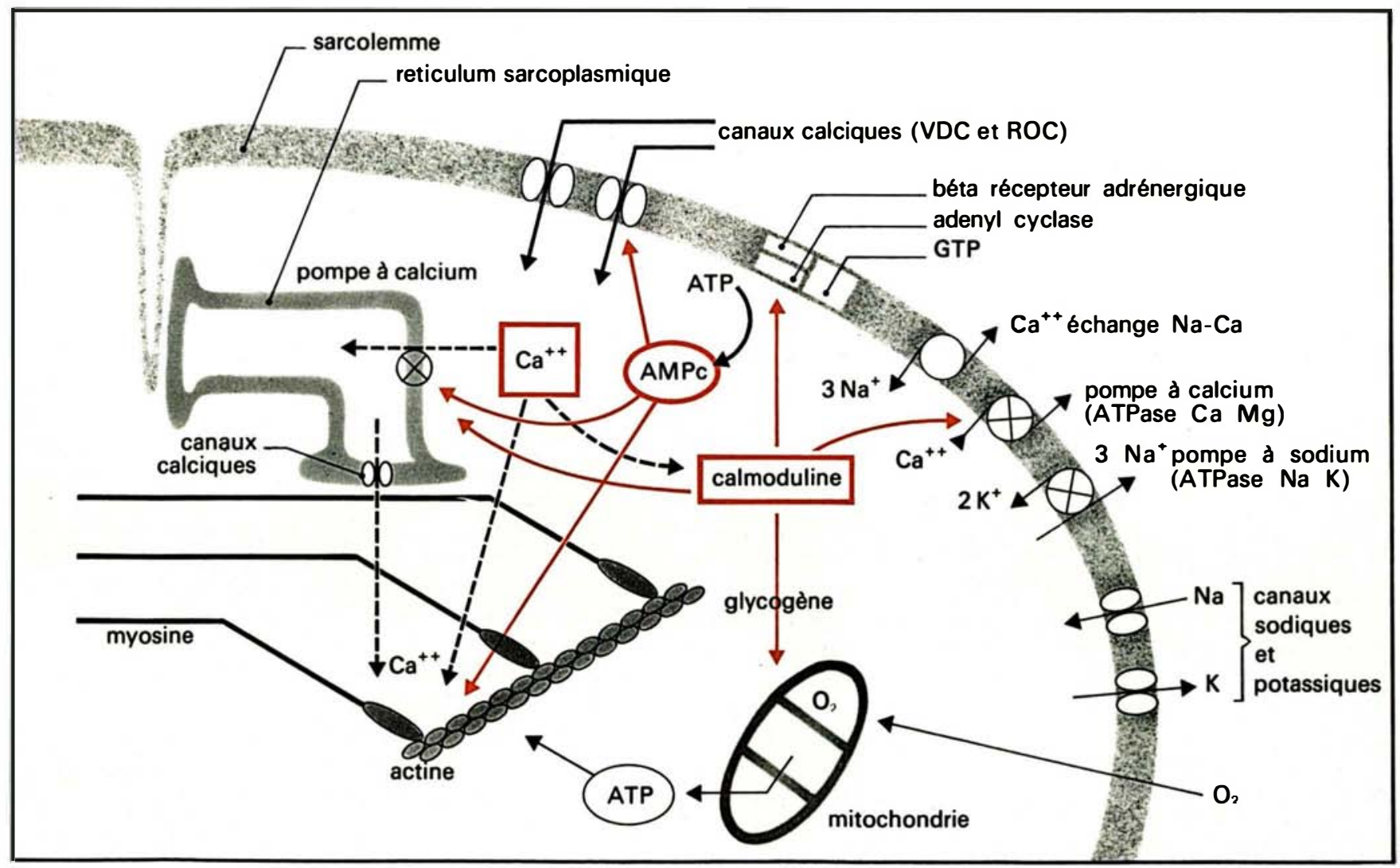




\section{REFERENCES}

I. Solaro RJ, Wise RM, Shiner JS, Briggs FN. Calcium requirements for cardiac myofibrillar activation. Circ Res 1974; 34: 525-30.

2. Fozzard HA, Beeler GW. Voltage clamp and cardiac electrophysiology. Circ Res 1975; 37: 403-13.

3. Morad M, Goldman YE, Trentham DR Rapid photochemical inactivation of $\mathrm{Ca}^{2+}$ antagonists shows that $\mathrm{Ca}^{2+}$ entry directly activates contraction in frog heart. Nature 1983; 304: 6358.

4. Ringer SA. Further contribution regarding the influence of the different constituants of the blood on the contraction of the heart. I Physiol (Lond) 1983; 4: 29-42.

5. Endo $M$, Tanaka $M$, Ogawa $Y$. Calcium induces calcium release of calcium from the sarcoplasmic reticulum of skinned skeletal niuscle fibers. Nature 1970; 228: 34-8.

6. Fabiato A. Fabiato F. Calcium and cardiac excitation-contraction coupling. Ann Rev Physiol I979; 41: 473-84.

7. Lullman H, Peters T. Action of cardiac glycoside on the excitation-contraction coupling in heart muscle. Prog Pharmacol 1978; 2: 3-53.

8. Brutsaert DL, Rademaker FE, Sys SU. Triple control of relaxation implications in cardiac disease. Circulation 1984; 69: 190-6.

9. Fabiato A. Effects of cyclic AMP and phosphodiesterase inhibitors on the contractile activation and the $\mathrm{Ca}^{++}$transient detected with aequorin in skinned cardiac cells from rat and rabbit ventricles. 7 Gen Physiol 1981; 78: 15-20.

10. Baker PF, Blaustein MP, Hodgkin AL, Steinhardt RA. The influence of calcium on sodium efflux in squid axons. 7 Physiol (Lond) 1969; 200: 431-58.

I I. Glitsche B, Reuter $H$. The effect of internal sodium concentration on calcium fluxes in isolated guinea pig auricles. 7 Physiol (Lond) 1970; 209: 25-43.

12. Lechat $\mathrm{Ph}$, Malloy $\mathrm{C}$, Smith TW. Active transport and inotropic state in guinea pig left atrium. Circ Res 1983; 52: 41 1-22.

13. Shelburne JC, Serena SD, Langer GA. Rate tension staircase in rabbit ventricular muscle. Relation to ionic exchange. Am 7 Physiol 1967 213: 111 5-24.

14. Artman M, Graham TP, Boucek RJ. Effects of post-natal maturation on myocardial contractile responses to calcium antagonists and changes in contraction frequency. 7 Cardiovasc Pharmacol $1985 ; 7: 850-5$. grâce d'une part aux pompes à calcium (ATPase $\mathrm{Ca}-\mathrm{Mg}$ dépendantes) et d'autre part grâce à un système d'échange $\mathrm{Na}-\mathrm{Ca}$ fonctionnant contre un gradient de concentration important. L'ATPase Ca-Mg dépendante est stimulée par la calmoduline qui augmente son affinité pour le calcium. Le système d'échange $\mathrm{Na}-\mathrm{Ca}$, initialement décrit au niveau des cellules nerveuses [ $\mathrm{I} 0$ ], puis au niveau cardiaque [I I] est électrogénique et expulse un calcium en échange de l'entrée de trois $\mathrm{Na}^{+}$. C'est le gradient sodique transmembranaire qui contrôle ce système d'échange : toute diminution du gradient sodique s'accompagne d'une augmentation du calcium intracellulaire par limitation de l'efflux calcique vers le milieu extracellulaire. Au maximum, l'échange peut s'inverser : expulser le sodium et faire entrer le calcium.

Les mécanismes de repompage calcique cytosolique (pompes du réticulum sarcoplasmique et du sarcolemme, échange $\mathrm{Na}-\mathrm{Ca}$ ) peuvent être dépassés lors d'un influx calcique massif (élévation du calcium extracellulaire, intoxication digitalique [12] (figures 3 et 4), ou bien lors de l'altération de l'un d'eux (ischémie); on aboutit alors au ralentissement de la relaxation puis à la contracture caractérisée par l'augmentation de la tension diastolique. A ce stade, la surcharge calcique cytosolique s'accompagne d'une acidose cellulaire qui est responsable d'une diminution de la tension systolique (figure 3).

\section{Relation tension- longueur du sarcomère}

Pour une concentration calcique intracellulaire donnée, la tension développée par le sarcomère dépend du nombre de ponts actine-myosine qui peuvent s'établir. Ce nombre dépend, entre autre, du degré initial de recouvrement des filaments d'actine et de myosine. Il est ainsi maximal pour une longueur de repos du sarcomère de 2 à $2,2 \mu$. Au-delà de $2,2 \mu$, la tension développée diminue par réduction du degré de recouvrement des filaments. En deça de $2 \mu$, la tension diminue : les filaments d'actine s'entrecroisent au milieu du sarcomère dans une zone où les têtes de la myosine sont moins nombreuses, fournissant donc moins de ponts actine-myosine. Il n'est pas exclu que d'autres mécanismes soient mis en jeu pour expliquer ces variations de tension avec la longueur du sarcomère, en particulier l'augmentation de l'affinité de la troponine $C$ pour le calcium lorsque la tension exercée sur la fibre augmente. Cette relation tension-longueur contribue au phénomène décrit par Starling : c'està-dire l'augmentation du travail ventriculaire parallèlement à une augmentation du volume de remplissage diastolique.

\section{Fréquence de stimulation}

Ce sont sur les préparations de cœur isolé in vitro que les influences de la fréquence de stimulation sur l'inotropisme peuvent être facilement mises en évidence. Ainsi sur l'oreillette isolée de cobaye, la tension développée décroît lorsque la fréquence de stimulation passe de 0,1 à I $\mathrm{Hz}$, passe par un minimum à I $\mathrm{Hz}$, puis augmente à nouveau pour des fréquences de I à $4 \mathrm{~Hz}$. C'est le staircase phenomenon. Audelà, on assiste à une relaxation incomplète avec élévation de la tension diastolique et diminution de la tension systolique. Une fois la période réfractaire atteinte, la fréquence de stimulation ne peut plus être augmentée.

Les caractéristiques du staircase phenomenon sont dépendantes des espèces considérées et des conditions expérimentales (température, concentration calcique du milieu). Plusieurs mécanismes peuvent être mis en jeu pour l'expliquer et le résultat dépend des cinétiques des différents systèmes de régulation des flux calciques. L'augmentation de la fréquence cardiaque augmente l'influx calcique transmembranaire au cours du potentiel d'action [13]. Plusieurs arguments plaident en faveur d'un rôle prépondérant du courant lent du potentiel d'action : le staircase positif est supprimé par le verapamil qui bloque le courant calcique [14]. Chez le rat adulte, le courant lent est très réduit; le staircase est négatif alors qu'il est positif chez le rat nouveau-né dont le courant lent du 


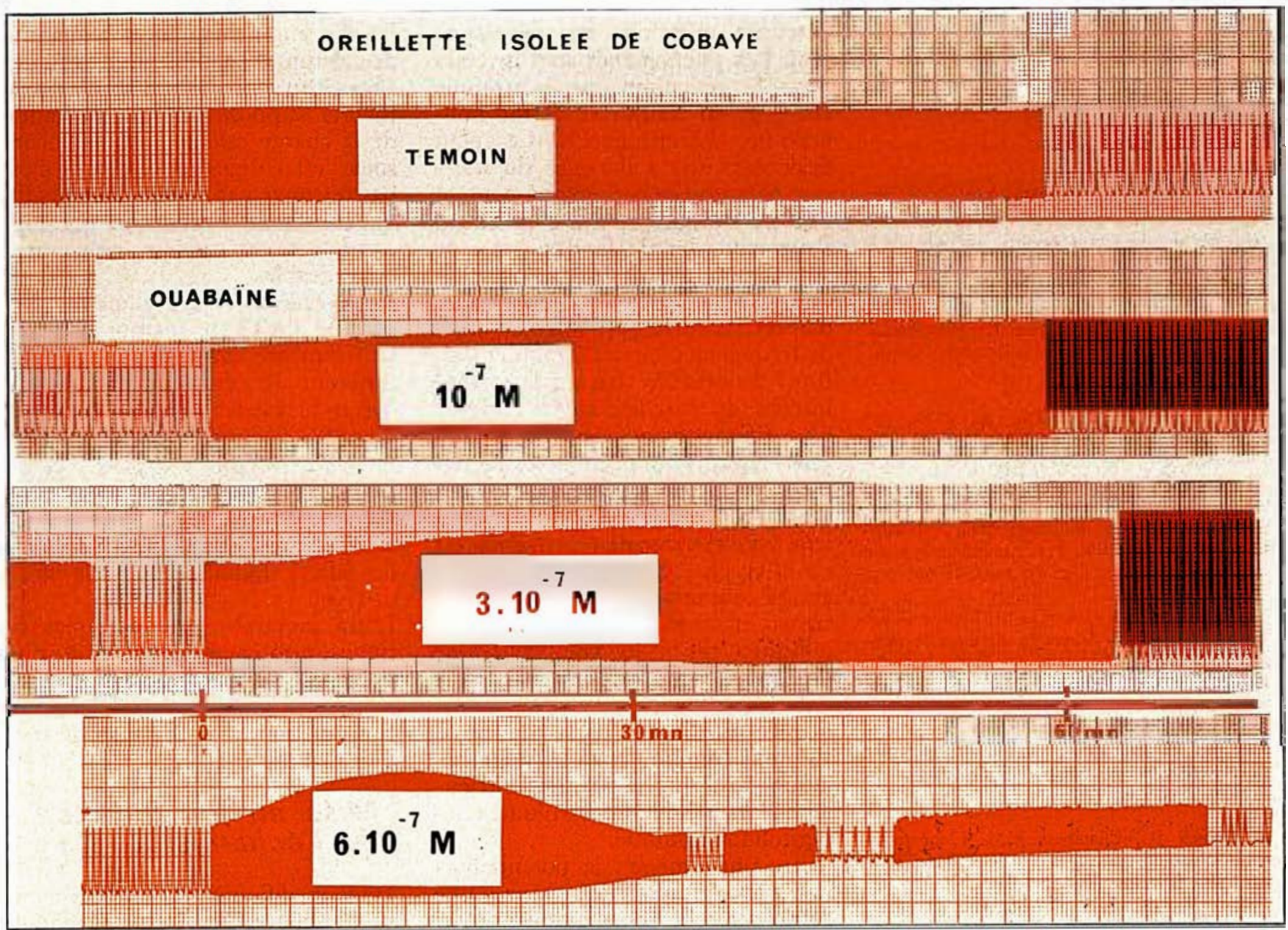

Figure 3. Effets inotropes digitaliques (ouabaïne) sur l'oreillette isolée de cobaye, à $30^{\circ}$ stimulée à $3,3 \mathrm{~Hz}$ avec une solution de Krebs contenant $1,8 \mathrm{mM}$ de Ca et $4 \mathrm{mM}$ de $K$. A partir de $6.10^{-7} \mathrm{M}$, l'effet inotrope s'accompagne d'une contracture (élévation de la tension diastolique par surcharge calcique cytosolique).

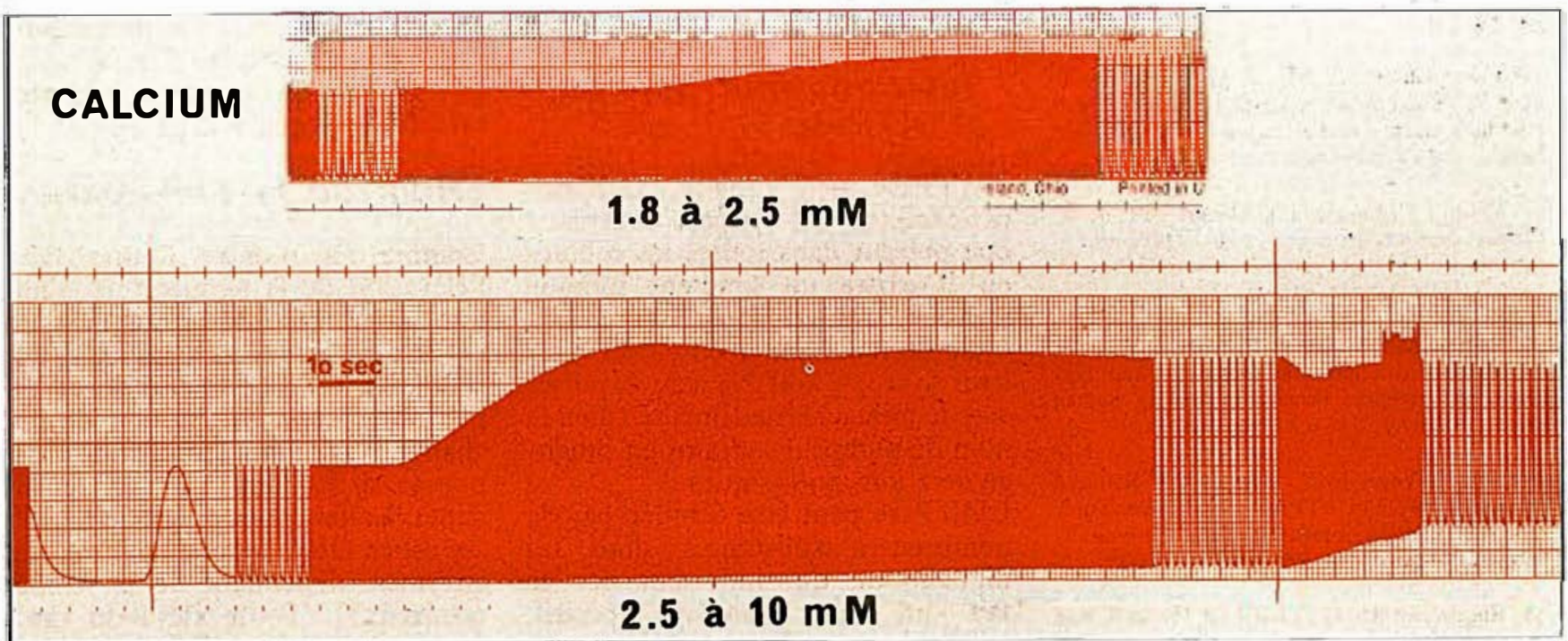

Figure 4. Effets inotropes induits par l'élévation de la concentration du calcium extra-cellulaire : en haut de 1,8 à 2,5 $m M$, en bas de 2,5 à Io $\mathrm{mM}$. A cette concentration apparaît une contracture traduisant le dépassement des mécanismes responsables de l'extrusion du calcium vers le milieu extra cellulaire (même préparation que figure 3 ).

$m / s n^{\circ} 2$ rol. 2 feurrier $\$ 6$ 


\section{REFERENCES}

15. Langer GA, Brady AJ, Tan ST, Serena SD Correlation of the glycoside response, the force staircase, and the action potential configuration in the neonatal rat heart. Circ Res 1975; 36: 744-52.

16. Schwartz A, Lindenmayer GE, Allen JC The sodium-potassium adenosine triphosphatase. Pharmacological and biochemical aspects. Pharmacol Rev 1975; 27: 3-134

17. Akera T, Brody T. The role of $\mathrm{Na}^{+} \mathrm{K}^{+}$ ATPase in the inotropic action of digitalis Pharmacol Rev 1978; 29: 187-220.

18. Wasserstrom JA, Schwartz DJ, Fozzard HA Relation between intracellular sodium and twitch tension in sheep cardiac Purkinje strands exposed to cardiac glycosides. Circ Res 1983; 51: 697-705.

19. Vassale $M$, Bhattacharyya $M$. Local anesthetics and the role of sodium in the force developped by canine ventricular muscle Purkinje fibers Circ Res 1980, 47: 666-74

20. Reuter H, Seitz N. The dependence of calcium influx from cardiac muscle in temperature and external ion composition. 7 Physiol (Lond) 1968; 195: 45I-70.

21. Russell JC, Chambers MM. Comparative temperature dependance of Na-K ATPase. Phy siol Chem Phys 1976; 8: 237-51.

22. Hoffman $B$, Lefkowitz RJ. Adrenergic receptors in the heart. Ann Rev Physiol 1982; 44: 47584.

23. Glossman M, Ferry DR, Lubbeck F, et al. Calcium channels: direct identification with radioligand binding studies. Trends $P$ harmacol $S c i$ 1982; 3: 431-7.

24. Lecarpentier YC, Martin JL, Gastineau P Hatt PY. Load dependence of mammalian hear relaxation during cardiac hypertrophy and heart failure. Am J Physiol 1982; 242: H855-61.

25. Cohn JN, Levine TB, Oliveri MT, et al Plasma norepinephrine as a guide to prognosis in patients with chronic congestive heart failure. $N$ Engl f Med 1984; 311: 819-23

26. Bristow MR, Ginsburg R, Minobe W, et al. Decreased cathecholamine sensitivity and beta adrenergic receptor density in failling human hearts. $N$ Engl 7 Med 1982; 307: 205-1 1.

27. Suko J, Vogel JMK, Chidsey CA. Reduced calcium uptake and ATPase of the sarcoplasmic reticular fraction prepared from chronically failing calf hearts. Circ Res 1970; 27: 235-47.

28. Engelmeier RS, O'Connell JB, Walsh R, Rad $\mathrm{N}$, Scanlon PJ, Gunnar RM. Improvement in symptoms and exercise tolerance by metoprolol in patients with dilated cardiomyopathy: a doubleblind, randomized, placebo-controlled trial. Circ potentiel d'action est développé [15]. Les phénomènes sont inversés pour le réticulum sarcoplasmique entre le rat adulte et le rat nouveau-né. L'échange $\mathrm{Na}-\mathrm{Ca}$ peut également être à l'origine du staircase phenomenon car, en augmentant l'influx sodique par unité de temps, l'augmentation de la fréquence de stimulation ralentit l'extrusion calcique par l'échange $\mathrm{Na}-\mathrm{Ca}$. Enfin, d'après Lullman [7] l'augmentation de fréquence pourrait diminuer l'affinité du calcium fixé sur le versant interne du sarcolemme à l'origine d'un eff et inotrope positif. Les staircase négatifs peuvent s'expliquer quand un potentiel d'action survient avant la réactivation du courant lent. Sa constante de réactivation est en effet plus grande que celle des autres courants et variable selon les tissus et espèces. Il est difficile d'évaluer in vivo chez l'homme l'importance du phénomène, mais le staircase est positif lorsque la fréquence cardiaque passe de 60 à I40/mn, comme l'atteste l'augmentation de $\mathrm{dp} / \mathrm{dt} \max$ intraventriculaire alors même que le volume télédiastolique diminue.

Autre phénomène : la potentialisation post-extrasystolique; après une extrasystole, le repos compensateur donne en effet au réticulum sarcoplasmique la possibilité de se recharger plus complètement en calcium, ce qui lui permet d'en délivrer une plus grande quantité au moment du cycle suivant.

\section{Relations inotropisme et ATPase}

L'ATPase membranaire Na-K-dépendante est un système enzymatique présent dans toutes les cellules où il existe un gradient ionique transmembranaire [16]. Au cours du cycle de fonctionnement, l'ATP est hydrolysée, 3 ions $\mathrm{Na}$ sont expulsés vers le milieu extracellulaire (d'où le nom de pompe à sodium) en échange de 2 ions potassiques.

L'ATPase peut être inhibée par de nombreuses substances dont les digitaliques, qui induisent par ce fait un effet inotrope positif. L'inhibition de la pompe à sodium induit en effet une élévation du $\mathrm{Na}$ intracellulaire, ce qui freine l'expulsion du calcium par l'échange
$\mathrm{Na}-\mathrm{Ca}$, augmentant ainsi la quantité de calcium disponible au niveau des sites contractiles $[17,18]$. Une telle théorie suppose une augmentation de la charge calcique intracellulaire sous l'effet digitalique, ce qui n'est pas retrouvé par certains auteurs [7] qui constatent au contraire une augmentation du calcium rapidement échangeable.

Le potassium extracellulaire $[\mathrm{Ke}]$ stimule l'ATPase membranaire en se fixant sur un site extracellulaire différent de celui des digitaliques. Toute élévation de $[\mathrm{Ke}]$ stimule la pompe à sodium et induit un effet inotrope négatif. De plus, l'élévation de $[\mathrm{Ke}]$ diminue la capacité de fixation des digitaliques sur l'ATPase; ceci explique la potentialisation des effets digitaliques par la chute de $[\mathrm{Ke}]$.

Enfin, certaines substances plasmatiques, non encore identifiées, inhiberaient l'ATPase membranaire. Leur rôle physiopathologique dans la régulation de la contractilité reste à déterminer.

\section{Mise en jeu directe de l'échange $\mathrm{Na}-\mathrm{Ca}$}

Toute modification du gradient transmembranaire [ $\mathrm{Nai}] /[\mathrm{Na}$ e] module directement l'échange $\mathrm{Na}-\mathrm{Ca}$. Toute élévation de [ $\mathrm{Na}$ i] ou toute baisse de $[\mathrm{Na}$ e] freine la sortie calcique et augmente la contractilité. C'est par un tel mécanisme que les substances antiarythmiques quinidiniques, en diminuant l'influx sodique du potentiel d'action, induisent un eff et inotrope négatif [19].

\section{Rôle de la température}

Comme l'a montré Reuter [20], l'élévation de la température diminue la contractilité en augmentant l'efflux calcique vers le milieu extracellulaire. L'un des mécanismes responsable est la diminution de l'échange $\mathrm{Na}-\mathrm{Ca}$ par stimulation de la pompe à sodium ATPase dépendante. Le fonctionnement de l'ATPase est en effet, comme pour tous les enzymes, dépendant de la température [2I]. Toute élévation thermique chez un insuffisant cardiaque peut, en déprimant la contractilité, favoriser la survenue d'un épisode de décompensation. 
L'influx calcique du courant lent du potentiel d'action se fait grâce à deux types de " canaux calciques " : les canaux voltage-dépendant (VDC: voltage-dependent channel) et ceux dépendant d'un récepteur hormonal (ROC: receptor operating channel).

\section{Modulation du courant calcique}

Ces derniers s'ouvrent à la suite de l'occupation des sites membranaires spécifiques pour les catécholamines (effets alpha), l'histamine ainsi que d'autres neurotransmetteurs [22]. Ces canaux sont insensibles aux antagonistes calciques. Par contre, les canaux calciques voltage-dépendant ont la capacité de fixer les différents antagonistes calciques qui induisent un effet inotrope négatif (figure 5). Plusieurs classifications ont été proposées : Glossman [23] distingue la classe I fixant les I-4 dihydropyridines, la classe II le Verapamil et la classe III le d-6-Diltiazem. A travers ces canaux voltage-dépendant, l'influx calcique peut être augmenté par l'AMP cyclique intracellulaire, par l'intermédiaire de la phosphorylation d'une protéine membranaire: la calciductine. La stimulation des récepteurs bêta-adrénergiques, en stimulant l'adenyl-cyclase, augmente le taux d'AMPc et induit un effet inotrope par l'augmentation du courant calcique. Les inhibiteurs des phosphodiestérases (caféine, amrinone) aboutissent au même résultat en freinant la dégradation de d'AMP cyclique. Enfin, par l'intermédiaire des récepteurs muscariniques et du GMP cyclique, les substances cholinergiques antagonisent les effets de l'AMP cyclique et diminuent la contractilité.

\section{Développement de l'insuffisance cardiaque}

Les mécanismes intimes à l'origine de l'altération de la contractilité qui est responsable du développement d'une insuffisance cardiaque sont encore inconnus.

Au cours des cardiopathies par surcharge barométrique (rétrécissement aortique, hypertension artérielle) ou volumétrique (insuffisance mitrale ou aortique, communication interventriçulaire), l'hypertrophie myocardique par synthèse de myofibrilles représente le principal phénomène compensateur initial tendant à rétablir un niveau normal de contrainte pariétale. Elle peut s'associer à des modifications isoenzymiques comme celle de l'ATPase de la myosine (voir l'article de $K$. Schmartz), modifications qui visent à améliorer le rendement. Dans les deux cas de surcharge de contrainte, après une période de compensation adaptée, l'apparition d'une dilatation cavitaire dans les surcharges barométriques, ou l'aggravation de la dilatation dans les surcharges volumétriques, traduit à la fois l'incapacité de l'hypertrophie compen-

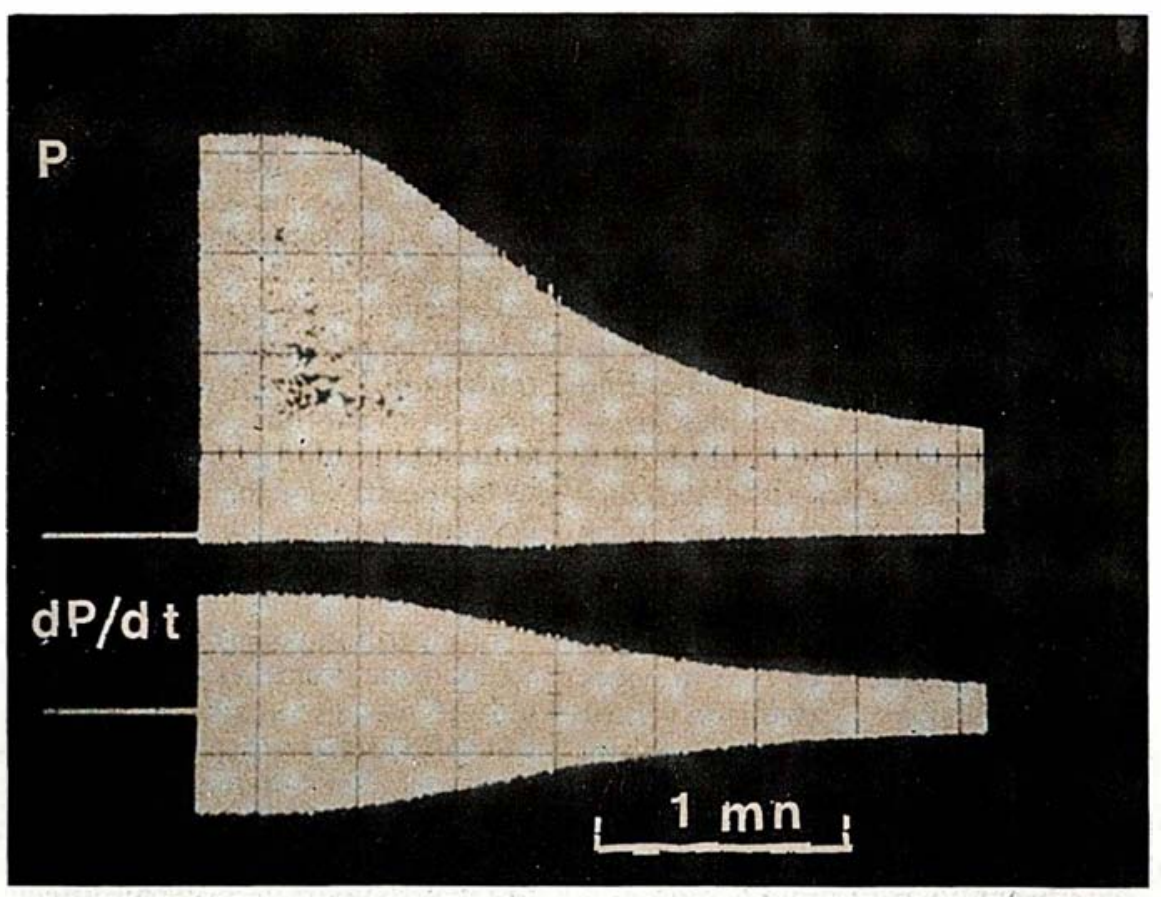

Figure 5. Effet inotrope négatif $d u$ verapamil $\mathrm{IO}^{-7} \mathrm{M}$, antagoniste calcique, sur une préparation de caur isolé de cobaye travaillant en isovolumétrie à $37^{\circ}$ et stimulé à 240/mn. En haut : la tension développée, en bas : $d p / d t$. 
satrice à maintenir la contrainte pariétale, mais surtout l'altération de la contractilité. On arrive beaucoup plus rapidement à ce stade de décompensation dans le cas des cardiomyopathies dilatées et de cardiopathies ischémiques. L'hypertrophie diminue la vitesse de relaxation mais la sensibilité de la relaxation à la charge n'est pas modifiée, même au stade d'insuffisance cardiaque [24].

D'autres mécanismes de compensation vont alors intervenir mais ceux-ci vont s'auto-limiter et engendrer un cercle vicieux d'aggravation de l'insuffisance cardiaque. En effet, si l'élévation du tonus sympathique [25] tend à augmenter la contractilité, elle voit son efficacité limitée par le phénomène de désensibilisation et de diminution du nombre des récepteurs bêta-adrénergiques [26]. En dépit de son effet inotrope, la tachycardie induite peut démasquer l'ischémie latente des fibres sous-endocardiques par diminution de la durée de la diastole. Quant à l'effet alpha-adrénergique vasculaire, s'il tend à restaurer une pression de perfusion tissulaire par vasoconstriction, il augmente par ailleurs les résistances vasculaires et donc la contrainte systolique ventriculaire. Il en est de même de la vasoconstriction induite par l'angiotensine et l'arginine vasopressine. La rétention hydrosodée induite par l'hyperaldostéronisme répond à la diminution de perfusion rénale. Elle aboutit à une élévation des pressions de remplissage, qui tend à augmenter la contractilité par distension cavitaire (effet Starling), mais induit dans un deuxième temps œdèmes pulmonaire et périphériques. En fait, le phénomène de Starling ne semble pas jouer de rôle au cours des distensions cavitaires chroniques, mais uniquement lors des distensions aiguës. La longueur du sarcomère du ventricule dilaté de façon chronique n'est pas augmentée, du moins expérimentalement.

Au stade d'altération de la contractilité, il n'y a pas de diminution du taux des phosphates riches en énergie (ATP) au niveau du sarcomère mais une diminution de la vitesse de recaptage du calcium par le réticulum sarcoplasmique, qui s'accompagne d'une déplétion des stocks calciques intracellulaires [27]. Dans certains modèles expérimentaux (ischémie, cardiomyopathie du hamster), l'accumulation du calcium intracellulaire (cytosolique en particulier) pourrait être à l'origine de l'alteration contractile. L'ischémie et l'augmentation de la contrainte pariétale tendent à augmenter le calcium cytosolique par réduction du recaptage calcique par le réticulum sarcoplasmique et par augmentation de l'affinité du calcium pour la troponine $\mathrm{C}$.

Une telle surcharge calcique cytosolique pourrait ainsi engendrer à long terme un processus de détérioration de la contractilité.

Les traitements de l'insuffisance cardiaque doivent donc s'attacher à réduire la contrainte pariétale et l'ischémie myocardique. Diurétiques et vasodilateurs ont jusqu'à maintenant fourni de bons résultats fonctionnels chez l'insuffisant cardiaque en réduisant la contrainte pariétale et donc, l'ischémie relative des couches sous-endocardiques, lorsqu'elle existe. L'efficacité des traitements visant à élever les concentrations calciques au niveau des sites contractiles reste problématique car ils sont potentiellement nocifs à long terme s'ils ne stimulent pas parallèllement le recaptage calcique par le réticulum sarcoplasmique. D'ou la nécessité de la correction d'une éventuelle ischémie associée qui ralentit le recaptage calcique par le réticulum sarcoplasmique. C'est peut-être par un tel mécanisme anti-ischémique que les bêta-bloquants ont permis d'obtenir des améliorations fonctionnelles et une augmentation de la fraction d'éjection, à priori paradoxales, en cas de cardiomyopathies [28]. L'effet inotrope négatif et la bradycardie induite par ces substances, diminue l'ischémie relative des couches myocardiques sous-endocardiques qui assurent la majorité du travail ventriculaire. Cette réduction de l'ischémie pourrait prévenir l'accumulation calcique cytosolique et ralentir, par ce mécanisme, l'altération de la contractilité. Quant à la question du meilleur traitement à administrer pour stabiliser une insuffisance cardiaque et empêcher la dégradation de la contractilité, elle reste posée

\section{Summary}

During the action potential, calcium enters the cardiac cell through the slow inward current. Contraction however requires a larger amount of calcium provided by mobilisation of intracellular calcium stores. Contraction develops when calcium is bound to troponin $\mathrm{C}$ which allows actin-myosin interaction. Relaxation occurs with reduction of cytosolic calcium concentration following inactivation of the calcium inward current and calcium uptake by the sarcoplasmic reticulum and sarcolemmal calcium pumps.

During diastole, intracellular ionic homeostasis is restored by the sodium pump (Na-KATPase), the calcium pump (Mg-Ca ATPase) and the $\mathrm{Na}-\mathrm{Ca}$ exchange. Physiologic variations of developped tension depend on modifications of cytosolic calcium concentrations occurring with alterations of sarcomere length, rate of stimulation and temperature. Moreover calcium fluxes are regulated through cAMP, calmodulin and protein kinases. Mechanisms of alteration of contractility leading to heart failure remain unknown. Deterioration however occurs when compensatory mechanisms can no longer restore a normal level of wall stress. Chronic cytosolic calcium overload especially during ischemia might represent a major adverse effect.

\section{TIRES A PART}

Ph. Lechat : Chu Pitié-Salpêtrière, service de cardiologie, 47 Boulevard de l'Hôpital, 75651 Paris Cedexi3. 